\title{
A flea and tick collar containing $10 \%$ imidacloprid and $4.5 \%$ flumethrin prevents flea transmission of Bartonella henselae in cats
}

\author{
Michael R Lappin ${ }^{1 *}$, Wendell L Davis ${ }^{2}$, Jennifer R Hawley ${ }^{1}$, Melissa Brewer ${ }^{1}$, Arianne Morris ${ }^{1}$ \\ and Dorothee Stanneck ${ }^{3}$
}

\begin{abstract}
Background: Bartonella henselae is transmitted amongst cats by Ctenocephalides felis and is associated with multiple clinical syndromes in cats and people. In a previous study, monthly spot-on administration of 10\% imidacloprid/1\% moxidectin was shown to block transmission of $B$. henselae amongst cats experimentally exposed to infected $C$. felis. The purpose of this study was to determine whether application of a flea and tick collar containing 10\% imidacloprid and $4.5 \%$ flumethrin would lessen C. felis transmission of B. henselae amongst cats for 8 months.

Methods: Specific pathogen free cats $(n=19)$ were housed in three adjoining enclosures that were separated by mesh to allow $C$. felis to pass among groups but prevent cats in different enclosures from contacting one another. One group of 4 cats was inoculated intravenously with $B$. henselae and after infection was confirmed in all cats based on positive PCR assay results, the cats were housed in the middle enclosure. The $B$. henselae infected cat group was flanked by a group of 8 cats that had the collar placed and maintained for the duration of the study and a group of 7 cats that were not treated. Ctenocephalides felis (50 males and 50 females) raised in an insectary were placed on each of the 4 cats in the $B$. henselae infected group monthly for 7 applications and then every 2 weeks for 4 applications starting the day the collar was applied. Blood was collected from all cats weekly for Bartonella spp. PCR, serology and culture.
\end{abstract}

Results: While side-effects associated with the collars were not noted, persistent fever necessitating enrofloxacin therapy occurred in two of the untreated cats. While $B$. henselae infection was ultimately confirmed in 4 of 7 of the untreated cats, none of the cats with collars became infected ( $P=0.026)$.

Conclusions: In this study design, use of a collar containing 10\% imidacloprid and $4.5 \%$ flumethrin was well tolerated and prevented C. felis transmission of B. henselae amongst cats for 8 months.

Keywords: Bartonella, Ctenocephalides, Imidacloprid

\section{Background}

Bartonella henselae is transmitted amongst cats by Ctenocephalides felis and is associated with multiple clinical syndromes in cats and people [1-7]. In addition, Bartonella koehlerae and Bartonella clarridgeiae have commonly been cultured or amplified from fleas, cats, and people. Being scratched by a young cat is a major

\footnotetext{
* Correspondence: mlappin@colostate.edu

${ }^{1}$ Department of Clinical Sciences, Center for Companion Animal Studies, Colorado State University, Fort Collins, USA

Full list of author information is available at the end of the article
}

risk factor for development of bartonellosis in people. Bartonella henselae is passed in C. felis frass and lives outside the body for 9 days or longer [8]. It is possible that the combination of $C$. felis and the grooming behavior of cats leads to Bartonella spp. contamination of claws. In one study of cats housed in shelters in Florida and Alabama with high risk for C. felis infestation, DNA of Bartonella spp. was amplified from the claws of 9 of 51 cats $(17.6 \%)$ [6].

Use of flea control products that lessen transmission of Bartonella spp. amongst cats by C. felis may indirectly 
lessen the risk of bartonellosis to humans. In a previous study, monthly spot-on administration of $10 \%$ imidacloprid/1\% moxidectin ${ }^{\mathrm{a}}$ was shown to block transmission of $B$. henselae amongst cats experimentally exposed to infected C. felis [7]. However, this product is licensed for monthly use and so owner compliance may be less than $100 \%$. A flea and tick collar containing $10 \%$ imidacloprid and $4.5 \%$ flumethrin ${ }^{\mathrm{b}}$ was recently registered in the European Union and the United States [9-11]. This collar has been shown to be safe for use in kittens $>10$ weeks of age and is very effective for the prevention of fleas and ticks for 8 months which could be very convenient for some cats and their owners [9-11].

The purpose of this study was to determine whether application of a flea and tick collar containing 10\% imidacloprid and $4.5 \%$ flumethrin to cats would lessen C. felis transmission of $B$. henselae amongst cats over an 8 month study period.

\section{Methods}

Bartonella henselae isolate

Bartonella henselae strain CSU-1 was initially isolated from a shelter cat in Florida and has been passaged once in cats [7]. Blood in EDTA from a cat infected with CSU-1 by exposure to infected $C$. felis had been stored at $-80^{\circ} \mathrm{C}$ until used in the current study.

\section{Ctenocephalides felis}

The C. felis used in this study were purchased from an insectary of a local research laboratory. ${ }^{\mathrm{C}}$ Two groups of 5 male and 5 female adult $C$. felis that were not exposed to the cats were purchased (one group before the study began and another group after the completion of this study) and were pooled by group, subjected to total DNA extraction, and shown to be negative for DNA of Bartonella spp. and haemoplasmas by PCR assay $[6,11,12]$.

\section{Flea and tick collar}

A flea and tick collar that contains imidacloprid 10\% and flumethrin $4.5 \%$ was placed on designated cats in this study following the manufacturer's guidelines [9-11]. ${ }^{\mathrm{b}}$ The collars were stored in a locked, fireproof cabinet and maintained at $20^{\circ} \mathrm{C}$ to $25^{\circ} \mathrm{C}$ until used.

\section{Animals}

A total of 19 cats (10 male; 9 female) were purchased from a commercial research cattery at approximately 4 months of age and shipped to the study site. The cats had been vaccinated against feline viral rhinotracheitis, feline calicivirus, and feline panleukopenia virus. During the 49 day equilibration period for the study, the male cats were neutered following protocols of the animal facility, all cats were shown to have negative serum test results for FeLVantigen, FIV-antibody, Dirofilaria immitis antigen, ${ }^{\mathrm{d}}$ and
Bartonella spp. antibody (ELISA), and all cats were shown to be negative for Bartonella spp and haemoplasma DNA in blood using previously reported PCR assays [12-14]. A temperature sensing microchip was implanted as previously described for use during clinical monitoring over the course of the study [15].

\section{Experimental design}

The study design was approved by the Institutional Animal Care and Use Committee at the research facility that housed the cats during the flea infestation portion of the study and the Animal Care and Use Committee at Bayer Animal Health.

After arrival at the facility and entry into the 49 day equilibration period, the cats were randomized into 3 groups and housed together in 1 room that was divided into 3 sections (R1, R2, and R3) beginning on Day -44 . The 3 sections were separated from each other by mesh so that $C$. felis could move amongst the cats, while body contact between cats or fighting with members of other groups was prevented. Sections R1 and R3 were adjacent to the center section R2 but not to each other. Approximately $25 \%$ of the floor space in each section was covered in carpet to promote survival and a complete life cycle of C. felis within the room. Cat perches were placed in the corners of each section adjacent to the mesh dividing the sections to encourage cats from the various groups to be close to each other. Based on this design, it seems unlikely the physical structure itself would influence C. felis to preferentially transfer to R1 or R3.

The group in $\mathrm{R} 1$ ( $\mathrm{n}=7$ cats) were untreated control cats throughout the study, the group in R2 ( $\mathrm{n}=4$ cats) were infected with $B$. henselae by intravenous inoculation of $0.2 \mathrm{ml}$ of blood containing $B$. henselae strain CSU-1 on Day - 39 of the study, and the flea and tick collars were placed on the group in R3 on Day 0 of the study. After placement of the collars on Day 0, a total of 50 males and 50 female $C$. felis were placed on each of the 4 cats in the B. henselae infected group in R2. Additional fleas (50 males and 50 female) were placed on each of the R2 cats monthly for an additional 6 applications and then every 12 - 14 days for 4 applications. The interval between $C$. felis application was shortened at the end of the experiment to increase likelihood of $B$. henselae infection in all cats. Flea counts by flea comb were determined every $12-14$ days for the duration of the study and the fleas returned to the cat. The flea collars were removed from R3 cats on Day 238 of the study, all cats were administered imidacloprid ${ }^{f}$ once at that time, and then were observed and sampled as described until Day 254. Once shown to be negative for B. henselae by PCR, the cats were returned to the research facility cat colony with biosafety committee approval. 
Samples were collected for performance of Bartonella spp. serology and PCR assay for amplification of Bartonella spp. DNA on Days 0, 14, 28, 42, 56, 70, 84, 98, 112, 126, $140,154,168,182,196,210,224,238$, and 252 for all cats as well as on Days $-30,-23,-16,-9$ and -2 for cats in R2.

\section{Assays}

Blood samples (2 $\mathrm{ml}$ in a clot tube for serum separation; $1 \mathrm{ml}$ in EDTA) from each cat were transported from the research facility to Colorado State University within 2 hours of collection. Samples were prepared for determination of serum titers of IgG against Bartonella spp. and for PCR assay for Bartonella spp. DNA on the day of collection $[12,14]$. One aliquot of each blood sample $(500 \mu \mathrm{L})$ in EDTA was stored at $-80^{\circ} \mathrm{C}$ for bacterial culture, pending results of the Bartonella spp. PCR assay. Samples negative for Bartonella spp. DNA by PCR assay were cultured using the stored blood as previously described [7]. Results for samples that yielded characteristic Bartonella spp. colonies were confirmed by Bartonella spp. PCR assay.

\section{Clinical monitoring}

The cats were examined daily for attitude and appetite throughout the study. Mucous membrane color assessment and physical examination (including cardiac auscultation) was made on any cat exhibiting signs of depression or inappetance and for cats with a rectal body temperature of $>102.5^{\circ} \mathrm{F}\left(39.2^{\circ} \mathrm{C}\right)$. Any cat with fever had a complete blood cell count performed. Cats that developed clinical illness consistent with bartonellosis (fever and inappetance of $>2$ days duration) during the study were administered enrofloxacin $^{\mathrm{g}}$ at $5 \mathrm{mg} / \mathrm{kg}, \mathrm{PO}$, daily for at least 14 days and supportive care as indicated. Cats requiring antibiotic treatment were administered imidacloprid ${ }^{f}$ and moved to a $C$. felis free room for continued care.

\section{Cat hair sampling}

On Day 224, approximately $0.5 \mathrm{~g}$ of hair was collected from each cat. Each sample was placed into an individually labelled plastic bag for determination of imidacloprid concentrations. ${ }^{\mathrm{h}}$ The cutoff sensitivity for the imidacloprid assay is $0.1 \mathrm{mg} / \mathrm{kg}$ hair.

\section{Statistical analysis}

Cats were diagnosed with $B$. henselae infection if at least 2 samples over the course of the study were positive for Bartonella spp. IgG, Bartonella spp. DNA by PCR assay, or Bartonella spp. organisms by culture. The proportion of $B$. henselae-infected cats was compared between groups R1 and R3 by use of a 2-tailed Fisher exact test. Differences among group mean flea counts were compared with the 2 -tailed Student $t$ test. A value of $P<0.05$ was considered significant for all analyses.

\section{Results}

\section{Clinical findings}

Side-effects associated with the collars were not noted in any cat throughout the study. While none of the cats in R2 or R3 developed clinical signs of bartonellosis after starting the $C$. felis infestations, 2 cats in R1 developed clinical signs necessitating treatment. For one cat from group R1, clinical signs were first detected on Day 47 of the study; the cat was normal 3 days after starting enrofloxacin therapy (14 days total) and fluids subcutaneously (once). The complete blood cell count for this cat was normal. The infection status of this cat was monitored in a separate room for several months. As the cat was still positive for B. henselae DNA on Day 154 and had only been administered imidacloprid once, over 100 days previously, it was returned to R1 to provide a continued source of $B$. henselae to attempt maximize the challenge infection for other cats in R1 and the cats in R3.

One other cat from group R1 developed clinical signs of bartonellosis on Day 124. Fever, lethargy, and a II/VI systolic left base heart murmur were the most significant findings. The complete blood cell count was normal other than the presence of 200 band neutrophils/ $\mu$ l (normal $=0 \mathrm{cell} / \mu \mathrm{l}$ ) which could suggest inflammation. A scanning echocardiogram showed normal valve leaflets and so the cardiac murmur was believed to be from stress. The cat was negative for Bartonella spp. IgG but positive for B. henselae DNA by PCR assay on Day 124. The fever and lethargy resolved over the first 3 days of enrofloxacin administration (28 days total) and the heart murmur was not noted on subsequent recheck examinations. The cat was positive for Bartonella DNA alone on Days 154 and positive for Bartonella DNA and IgG on Day 168 and Day 182. This cat was not returned to the study.

\section{Assay results}

All 4 of the group R2 cats became seropositive and PCR assay positive after IV inoculation. Bartonella henselae DNA was amplified from all 4 cats from Day $0-28$ of the study. From Day 42 to Day 168, 1 - 3 cats in R2 were PCR positive on any given day. All R2 cats were PCR negative from Day 182 to Day 252. Each of the R2 cats seroconverted with maximal titers of $64 ; 128 ; 512$; and 1028. The first positive antibody test results were detected on Day 56; Day 154 (2 cats), and Day 168. All 4 R2 cats were still seropositive on Day 252 (titers $=64$; $64 ; 128 ; 256)$.

Of the 7 cats in R1 (no treatment), 4 cats had $B$. henselae amplified from blood with the first positive test results being detected on Days 28, 42, 126, and 140 (Figure 1). The first positive antibody test results for R1 cats were detected on Day 56; Day 154 (2 cats), and Day 168. Maximal titers were 64; $128 ; 512$; and 4,096. None of the PCR negative cats were blood culture positive. 


Days after initial C. felis placement
\begin{tabular}{|c|c|c|c|c|c|c|c|c|c|c|c|c|c|c|c|c|c|c|c|}
\hline Cat & $\mathbf{0}$ & $\mathbf{1 4}$ & $\mathbf{2 8}$ & $\mathbf{4 2}$ & $\mathbf{5 6}$ & $\mathbf{7 0}$ & $\mathbf{8 4}$ & $\mathbf{9 8}$ & $\mathbf{1 1 2}$ & $\mathbf{1 2 6}$ & $\mathbf{1 4 0}$ & $\mathbf{1 5 4}$ & $\mathbf{1 6 8}$ & $\mathbf{1 8 2}$ & $\mathbf{1 9 6}$ & $\mathbf{2 1 0}$ & $\mathbf{2 2 4}$ & $\mathbf{2 3 8}$ & $\mathbf{2 5 2}$ \\
\hline 1 & 0 & 0 & 0 & 0 & 0 & 0 & 0 & 0 & 0 & 0 & 0 & 0 & 128 & $\mathbf{2 2}$ & & & & & \\
\hline 2 & 0 & 0 & 0 & 0 & 0 & 0 & 0 & 0 & 0 & 0 & 0 & 0 & 0 & 0 & 0 & 0 & 0 & 0 & 0 \\
\hline 3 & 0 & 0 & 0 & 0 & 0 & 0 & 0 & 0 & 0 & 0 & 0 & 64 & 64 & 64 & 64 & 0 & 64 & 128 & 64 \\
\hline 4 & 0 & 0 & 0 & 0 & 64 & 128 & 128 & 512 & 128 & 256 & 256 & 512 & 256 & 256 & 512 & 128 & 256 & 256 & 256 \\
\hline 5 & 0 & 0 & 0 & 0 & & & & & & & & 4096 & 1024 & 1024 & 1024 & 4096 & 512 & 512 & 512 \\
\hline 6 & 0 & 0 & 0 & 0 & 0 & 0 & 0 & 0 & 0 & 0 & 0 & 0 & 0 & 0 & 0 & 0 & 0 & 0 & 0 \\
\hline 7 & 0 & 0 & 0 & 0 & 0 & 0 & 0 & 0 & 0 & 0 & 0 & 0 & 0 & 0 & 0 & 0 & 0 & 0 & 0 \\
\hline
\end{tabular}

Figure 1 Bartonella henselae antibody and PCR assay results in 7 untreated cats (R1) exposed to $C$. felis allowed to feed on cats with $B$. henselae infection induced by IV inoculation. Light gray shaded areas indicate samples that were PCR positive; Dark gray areas indicate the days on which the cats were removed from the room because of fever and need for supportive care; exposure to $C$. felis began on Day 0 . None of the PCR negative cats were blood culture positive.

While $B$. henselae infection was ultimately confirmed in 4 of 7 of the untreated cats, none of the R3 cats with collars became infected $(\mathrm{P}=0.026)$.

\section{Ctenocephalides felis counts}

Most group R2 cats had detectable infestations when C. felis counts were made over time (Figure 2). However, the number of $C$. felis per cat varied on a given day. Only one $C$. felis was found on one cat in R3 over the course of the study (Day 238). The C. felis counts were variable for the cats in R1 as well. The only day that C. felis counts were significantly different amongst groups was on Day 14 when cats in R2 had great numbers of C. felis than cats in R1 or R3.

\section{Cat hair sampling}

Imidacloprid could be detected in the hair samples of all group R3 cats, some of the R1 cats (4 of 6) and some of the R2 cats (1 of 4). The values varied amongst group R1 (mean $=0.25 \mathrm{mg} / \mathrm{kg}$ hair; $\mathrm{SD}=0.22$; range $=0-0.62$ ), $\mathrm{R} 2$ (mean $=0.04 \mathrm{mg} / \mathrm{kg}$ hair; $\mathrm{SD}=0.07$; range $=0-0.15$ ), and R3 $($ mean $=32.4 \mathrm{mg} / \mathrm{kg}$ hair; $\mathrm{SD}=7.93$; range $=17.89-$ 41.71).

\section{Discussion}

In this setting, use of a collar containing 10\% imidacloprid and $4.5 \%$ flumethrin was well tolerated and prevented C. felis transmission of this strain of $B$. henselae amongst cats. These results are similar to those achieved in a

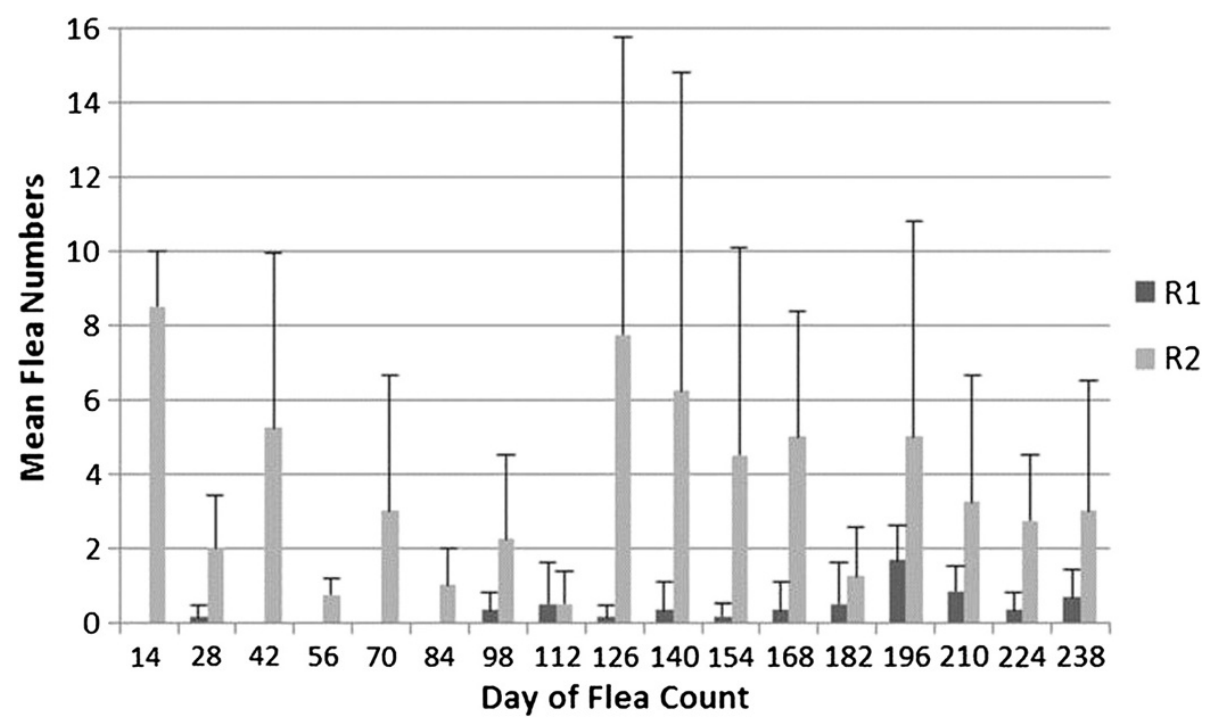

Figure 2 Results (mean and standard deviation) of Ctenocephalides felis counts over time after placement of a flea collar on cats housed in R3 which was adjacent to R1 and R2. The 4 cats in R2 had been experimentally infected with Bartonella henselae and a total of 50 males and 50 female C felis were placed on the R2 cats on Day 0, monthly for an additional 6 applications and then every 12 - 14 days for 4 applications. There were $6-7$ cats in R1 over the course of the study. Only one C. felis was found on one cat in R3 over the course of the study (Day 238). The only day that C. felis counts were significantly different amongst groups was on Day 14 when cats in R2 had greater numbers of C. felis than cats in R1 or R3. 
previous study using 10\% imidacloprid/1\% moxidectin applied topically every month [7]. A potential limitation of the current study was the relatively low flea burdens on the cats in R1 detected over the course of the study which may have lessened the potential for transmission of $B$. henselae among the cats in this group. In the previous study, $100 \%$ of the control group developed $B$. henselae infection within the 3 month study period in contrast to a $57.1 \%$ infection reported here. The relatively low flea numbers on cats in R1 and R2 may also have lessened the risk for acquiring $B$. henselae infection in the cats with collars in R3.

The previous study of $10 \%$ imidacloprid/1\% moxidectin applied topically every month used essentially the same study design [7]. However, in that study mean C. felis numbers on the control cats were generally $>2$ fleas/cat after the first 14 days of the study which was numerically higher than the $C$. felis numbers reported in the control cats (R1) described here. Day 14 was the only time of the study described here that showed statistically higher C. felis counts in cats housed on R2 when compared to those in R1 and R3. This finding persisted even when the C. felis infestations were increased to every 14 days for the last month of the study. One likely explanation for these findings is that imidacloprid was shared amongst cats in the three groups by contact through the mesh walls. This hypothesis was the reason for collection of hair samples at day 224 for evaluation of their imidacloprid content. As imidacloprid is a very efficacious active ingredient, even low doses could interfere with flea survival on the animals and via killing flea larvae also interrupt the establishment of a constant flea population. Imidacloprid values found in the treated cat's hair fit to the values detected in prelicensing studies (Unpublished Data, 2012). However, traces of imidacloprid being at up to approximately $1 \%$ of this amount were also detected in the hair samples of untreated cats from R1 and R2. While only low concentrations of imidacloprid were detected, this was likely enough to lessen $C$. felis numbers, especially by interfering with the flea development life cycle.

At least one cat was B. henselae infected until Day 210 of the study and so could have served as a source of B. henselae for the new C. felis added in the later weeks of the experiment. These results suggest that the collar prevented $B$. henselae infection to the cats in R3 for the entire 8 month study period.

Development of fever and lethargy in 2 of 7 cats (28.5\%) was similar to what was reported in the previous $10 \%$ imidacloprid/1\% moxidectin study [7]. The results suggest that the CSU-1 strain of $B$. henselae is pathogenic to some cats and that virulence was not changed by one passage through cats. As observed in the previous study, the IV inoculated cats in R2 did not develop clinical illness which suggests pathogenic factors may be provided by the vector
[7]. The two clinically ill cats administered enrofloxacin had rapid clinical responses suggesting a treatment effect. However, bacteremia was not eliminated in cats administered either a 14 day or 28 day course of therapy. Future research on how to limit $B$. henselae bacteremia in cats is needed.

\section{Conclusions}

Use of imidacloprid containing products in this model can block transmission of this strain of $B$. henselae among cats. The CSU-1 strain of $B$. henselae can cause clinical disease in cats, particularly when vectored by C. felis.

\section{Endnotes}

aAdvantage Multi, Bayer HealthCare LLC, Shawnee Mission, KS;

${ }^{\mathrm{b}}$ Seresto Bayer Animal Health, Leverkusen, Germany; ${ }^{\mathrm{C}}$ HESKA Corporation, Loveland, CO;

${ }^{\mathrm{d}}$ SNAP ${ }^{\circledR}$ Triple, IDEXX Laboratories, Portland, ME; ${ }^{\mathrm{e}}$ High Quality Research, Fort Collins, CO;

${ }^{f}$ Advantage, Bayer HealthCare LLC, Shawnee Mission, KS; ${ }^{\mathrm{g} B a y t r i l, ~ B a y e r}$ HealthCare LLC, Shawnee Mission, KS; hayer Animal Health GmbH, Leverkusen, Germany.

\section{Abbreviations}

PCR: Polymerase chain reaction; $R$ 1: Room 1; R2: Room 2; R3: Room 3; SD: Standard deviation.

\section{Competing interests}

While this was a Bayer Animal Health funded project, all aspects were performed under GLP and GCP like conditions.

\section{Authors' contribution}

$M R L$ served as the consultant for the project, provided the infectious disease assays, was the interfacing person between the outside research facility and Bayer Animal Health, and wrote the first draft of the experimental design and the manuscript. DS at Bayer Animal Health provided the funding for the project, provided input on all stages of the project including the experimental design and manuscript, and provided the interface among Bayer Animal Health, the research facility, and Colorado State University. JRH, $M B$, and $A M$ provided scientific input into the study design and performed all assays. All authors approved the final version of the manuscript.

\section{Author details}

${ }^{1}$ Department of Clinical Sciences, Center for Companion Animal Studies, Colorado State University, Fort Collins, USA. ${ }^{2}$ Bayer HealthCare LLC, Shawnee, KS, USA. ${ }^{3}$ Bayer Animal Health GmbH, Leverkusen, Germany.

Received: 25 August 2012 Accepted: 21 December 2012 Published: 25 January 2013

\section{References}

1. Breitschwerdt EB, Maggi RG, Chomel BB, Lappin MR: Bartonellosis: an emerging infectious disease of zoonotic importance to animals and human beings. J Vet Emerg Crit Care 2010, 20:8-30.

2. Chomel BB, Boulouis HJ, Breitschwerdt EB: Cat scratch disease and other zoonotic Bartonella infections. J Am Vet Med Assoc 2004, 224:1270-1279.

3. Breitschwerdt EB, Maggi RG, Duncan AW, Nicholson WL, Hegarty BC, Woods CW: Bartonella species in blood of immunocompetent persons with animal and arthropod contact. Emerg Infect Dis 2007, 13:938-941.

4. Chomel BB, Kasten RW, Floyd-Hawkins K, Chi B, Yamamoto K, RobertsWilson J, Gurfield AN, Abbott RC, Pedersen NC, Koehler JE: Experimental transmission of Bartonella henselae by the cat flea. J Clin Microbiol 1996, 34:1952-1956. 
5. Lappin MR, Griffin B, Brunt J, Riley A, Burney D, Hawley J, Brewer MM, Jensen WA: Prevalence of Bartonella species, haemoplasma species, Ehrlichia species, Anaplasma phagocytophilum, and Neorickettsia risticii DNA in the blood of cats and their fleas in the United States. J Feline Med Surg 2006, 8:85-90.

6. Lappin MR, Hawley J: Presence of Bartonella species and Rickettsia species DNA in the blood, oral cavity, skin and claw beds of cats in the United States. Vet Dermatol 2009, 20:509-514.

7. Bradbury CA, Lappin MR: Evaluation of topical application of $10 \%$ imidacloprid-1\% moxidectin to prevent Bartonella henselae transmission from cat fleas. J Am Vet Med Assoc 2010, 236:869-873.

8. Higgins JA, Radulovic S, Jaworski DC, Azad AF: Acquisition of the cat scratch disease agent Bartonella henselae by cat fleas (Siphonaptera: Pulicidae). J Med Entomol 1996, 33:490-495.

9. Stanneck D, Rass J, Radeloff I, Kruedewagen E, Le Sueur C, Hellmann K, Krieger K: Evaluation of the long-term efficacy and safety of an imidacloprid 10\% / flumethrin $4.5 \%$ polymer matrix collar (Seresto (R)) in dogs and cats naturally infested with fleas and/or ticks in multicentre clinical field studies in Europe. Parasit Vectors 2012, 5:66

10. Stanneck D, Kruedewagen EM, Fourie JJ, Horak IG, Davis W, Krieger KJ: Efficacy of an imidacloprid/flumethrin collar against fleas and ticks on cats. Parasit Vectors 2012, 5:82. doi:10.1186/1756-3305-5-82.

11. Fourie JJ, Crafford D, Horak IG, Stanneck D: Prophylactic treatment of fleainfested cats with an imidacloprid/flumethrin collar to forestall infection with Dipylidium caninum. Parasit Vectors. 2012, 5:151 doi:10.1186/1756-3305-5-151

12. Jensen WA, Fall MZ, Rooney J, Kordick DL, Breitschwerdt EB: Rapid identification and differentiation of Bartonella species using a single-step PCR Assay. J Clin Microbiol 2000, 38:1717-1722.

13. Jensen WA, Lappin MR, Kamkar S, Reagan W: Use of a polymerase chain reaction assay to detect and differentiate two strains of Haemobartonella felis in naturally infected cats. Am J Vet Res 2001, 62:604-608.

14. Lappin MR, Breitschwerdt E, Brewer M, Hawley J, Hegarty B: Prevalence of Bartonella species DNA in the blood of cats with and without fever. J Fel Med Surg 2009, 11:141-148.

15. Quimby JM, Olea-Popelka F, Lappin MR: Comparison of digital rectal and microchip transponder thermometry in cats. J Am Assoc Lab Anim Sci 2009, 48:402-404.

doi:10.1186/1756-3305-6-26

Cite this article as: Lappin et al:: A flea and tick collar containing 10\% imidacloprid and $4.5 \%$ flumethrin prevents flea transmission of Bartonella henselae in cats. Parasites \& Vectors 2013 6:26.

\section{Submit your next manuscript to BioMed Central and take full advantage of:}

- Convenient online submission

- Thorough peer review

- No space constraints or color figure charges

- Immediate publication on acceptance

- Inclusion in PubMed, CAS, Scopus and Google Scholar

- Research which is freely available for redistribution

Submit your manuscript at www.biomedcentral.com/submit
C Biomed Central 